\title{
INVOLUTIONS IN SEMI-QUATERNIONS
}

\author{
MURAT BEKAR AND YUSUF YAYLI
}

Communicated by Abraham Ungar

Abstract. Involutions are self-inverse and homomorphic linear mappings. Rotations, reflections and rigid-body (screw) motions in three-dimensional Euclidean space $\mathbb{R}^{3}$ can be represented by involution mappings obtained by quaternions. For example, a reflection of a vector in a plane can be represented by an involution mapping obtained by real-quaternions, while a reflection of a line about a line can be represented by an involution mapping obtained by dual-quaternions. In this paper, we will consider two involution mappings obtained by semi-quternions, and a geometric interpretation of each as a planar-motion in $\mathbb{R}^{3}$.

MSC: 11R52, 53A25, 53A35, 70B10

Keywords: Dual-quaternions, involutions, planar-motion, semi-quaternions

\section{Introduction}

The adventure of quaternions started in the mid- $19^{\text {th }}$ century as a geometric and algebraic interest. Soon after they were found to have applications in mechanics, physics, computer graphic technology, mixed and augmented systems, etc. The main difficulty in the development of quaternions occured while defining the multiplication rule. Rumor says that the Irish mathematician Sir William Rowan Hamilton was looking for a way to formalize points in three-space in the same way that points in the plane can be defined in the complex field. For many years, he knew how to add and subtract points in three-space. However, he had failed by the problem of multiplication for over ten years. Finally, on 16 October 1843 in Dublin, Hamilton solved the multiplication problem and his intuition was that the algebra of quaternions would require three imaginary parts satisfying

$$
\boldsymbol{i}^{2}=\boldsymbol{j}^{2}=\boldsymbol{k}^{2}=\boldsymbol{i j} \boldsymbol{k}=-1 .
$$

Quaternions are useful tools for representing rotations, reflections and rigid-body (screw) motions in three-dimensional spaces. Ell and Sangwine [5] represented an involution and an anti-involution mapping of real-quaternions with their geometrical meanings as reflections or rotations in three-dimensional Euclidean space $\mathbb{R}^{3}$. 
Also, Bekar and Yayli [2-4] studied (anti)-involution mappings of complexifiedand dual split-quaternions with their geometrical meanings. In this paper, we will represent two mappings (one corresponding to a semi-quaternion involution and one to an anti-involution) and a geometric interpretation of each as a planar-motion in $\mathbb{R}^{3}$.

\section{Preliminaries}

Let $\mathrm{R}$ be a commutative ring and $f$ be a homomorphism (respectively anti-homomorphism) of an arbitrary $\mathrm{R}$-algebra $A$. Thus, $f: A \rightarrow A$ is $\mathrm{R}$-linear and

$f(1)=1$ and $f(a b)=f(a) f(b)$ (respectively $f(a b)=f(b) f(a)$ ) for all $a, b \in A$.

If $f$ is a homomorphism or anti-homomorphism of R-algebra such that $f^{2}=\operatorname{id}_{A}$, then $f$ is injective and surjective, and thus is an involution on $A$. An involution is called anti-involution if it is an anti-homomorphism. Assume throughout that $A$ is faithful that is if $r a=0$ for all $a \in A$ and some $r \in \mathrm{R}$, then $r=0$. Since the assignment $r \mapsto r 1_{A}$ injects $\mathrm{R} \hookrightarrow A$, we consider $\mathrm{R} \subseteq A$. Let $g$ be an antiinvolution on $A$ and define the norm and trace of $A$ by, respectively

$$
\mathrm{N}(a)=a(g(a)) \quad \text { and } \quad \operatorname{Tr}(a)=a+g(a) \quad \text { for all } \quad a \in A
$$

then $g$ is called standart-involution if $\mathrm{N}(a) \in \mathrm{R}$, see [7].

The set of real-quaternions can be given as

$$
\mathbb{H}=\left\{q=q_{0}+q_{1} \boldsymbol{i}+q_{2} \boldsymbol{j}+q_{3} \boldsymbol{k} ; q_{0}, q_{1}, q_{2}, q_{3} \in \mathbb{R}\right\}
$$

where the basis elements $\boldsymbol{i}, \boldsymbol{j}, \boldsymbol{k}$ satisfy the non-commutative multiplication rules

$$
\boldsymbol{i}^{2}=\boldsymbol{j}^{2}=\boldsymbol{k}^{2}=\boldsymbol{i} \boldsymbol{j} \boldsymbol{k}=-1, \quad \boldsymbol{i j}=-\boldsymbol{j} \boldsymbol{i}=\boldsymbol{k}, \quad \boldsymbol{j} \boldsymbol{k}=-\boldsymbol{k} \boldsymbol{j}=\boldsymbol{i}, \quad \boldsymbol{k} \boldsymbol{i}=-\boldsymbol{i} \boldsymbol{k}=\boldsymbol{j} .
$$

It is convenient to introduce $S_{q}=q_{0}$ and $\boldsymbol{V}_{q}=q_{1} \boldsymbol{i}+q_{2} \boldsymbol{j}+q_{3} \boldsymbol{k}$ which are called, respectively, the scalar and vector parts of $q=q_{0}+q_{1} \boldsymbol{i}+q_{2} \boldsymbol{j}+q_{3} \boldsymbol{k}$. If $S_{q}=0$, then $q=\boldsymbol{V}_{q}$ is said to be a pure and will be denoted by the boldface letter $\boldsymbol{q}$.

The multiplication of real-quaternions $q=S_{q}+\boldsymbol{V}_{q}$ and $p=S_{p}+\boldsymbol{V}_{p}$ is

$$
q p=S_{q} S_{p}-\left\langle\boldsymbol{V}_{q}, \boldsymbol{V}_{p}\right\rangle+S_{q} \boldsymbol{V}_{p}+S_{p} \boldsymbol{V}_{q}+\boldsymbol{V}_{q} \times \boldsymbol{V}_{p}
$$

where $S_{q}=q_{0}, S_{p}=p_{0}, \boldsymbol{V}_{q}=q_{1} \boldsymbol{i}+q_{2} \boldsymbol{j}+q_{3} \boldsymbol{k}, \boldsymbol{V}_{p}=p_{1} \boldsymbol{i}+p_{2} \boldsymbol{j}+p_{3} \boldsymbol{k}$. Also, $\left\langle\boldsymbol{V}_{q}, \boldsymbol{V}_{p}\right\rangle=q_{1} p_{1}+q_{2} p_{2}+q_{3} p_{3}$ and $\boldsymbol{V}_{q} \times \boldsymbol{V}_{p}=\left(q_{2} p_{3}-q_{3} p_{2}\right) \boldsymbol{i}+\left(q_{3} p_{1}-q_{1} p_{3}\right) \boldsymbol{j}+$ $\left(q_{1} p_{2}-q_{2} p_{1}\right) \boldsymbol{k}$ denotes, respectively, the usual inner and vector products of $\boldsymbol{V}_{q}$ and $\boldsymbol{V}_{p}$ in $\mathbb{R}^{3}$. 
Let $\mathbb{R}^{p, q, r}$ be the real vector space $\mathbb{R}^{p+q+r}$ with an orthogonal basis $\left\{e_{1}, e_{2}, \ldots, e_{n}\right\}$ equipped with a quadratic form $\mathrm{Q}$ as $\mathrm{Q}\left(e_{i}\right)=1$ for $1 \leq i \leq p, \mathrm{Q}\left(e_{i}\right)=-1$ for $p+1 \leq i \leq p+q$ and $\mathrm{Q}\left(e_{i}\right)=0$ for $p+q+1 \leq i \leq p+q+r . \mathrm{Cl}_{p, q, r}$ is the Clifford algebra generated by these basis vectors. Also, the space $\mathbb{R}^{p, q, 0}$ is denoted by $\mathbb{R}^{p, q}$ while the associated Clifford algebra being denoted by $\mathrm{Cl}_{p, q}$, see [12]. The algebra $\mathbb{H}$ is isomorphic to the Clifford algebra $\mathrm{Cl}_{0,2}$ (i.e., $\mathbb{H} \cong \mathrm{Cl}_{0,2}$ ) in dimension two where the standard anti-commuting generators $e_{1}, e_{2}$ satisfy

$$
e_{1}^{2}=e_{2}^{2}=\left(e_{1} e_{2}\right)^{2}=-1 \quad \text { and } \quad e_{1} e_{2}=-e_{2} e_{1}
$$

when the quaternionic units $\boldsymbol{i}, \boldsymbol{j}, \boldsymbol{k}$ are defined with, respectively, $e_{1}, e_{2}, e_{12}\left(=e_{1} e_{2}\right)$ in $\mathrm{Cl}_{0,2}$. Thus, multiplication in $\mathbb{H}$ is associative.

The quaternionic-conjugation of a real-quaternion $q=q_{0}+q_{1} \boldsymbol{i}+q_{2} \boldsymbol{j}+q_{3} \boldsymbol{k}$ is the standard involution $\bar{q}=q_{0}-q_{1} \boldsymbol{i}-q_{2} \boldsymbol{j}-q_{3} \boldsymbol{k}$, which amounts to the canonical involution of $\mathbb{H}$ as the Clifford algebra $\mathrm{Cl}_{0,2}$, and the norm of $q$ is

$$
\mathrm{N}(q)=q \bar{q}=\bar{q} q=q_{0}^{2}+q_{1}^{2}+q_{2}^{2}+q_{3}^{2} \in \mathbb{R} .
$$

If $\mathrm{N}(q)=1$, then $q$ is said to be a unit quaternion.

It should be noted that the concept norm of a quaternion does not match the concept Euclidean norm in linear algebra. For instance, the norm $\mathrm{N}(q)$ of a real-quaternion $q$ does not satisfy always the triangle inequality axiom of the Euclidean norm. Also, the norm of a dual-quaternion given by the Equation (5), does not always satisfy the positivity and triangle inequality axioms and it does not need to be real valued.

The multiplicative-inverse of a real-quaternion $q$ is

$$
q^{-1}=\frac{\bar{q}}{\mathrm{~N}(q)}, \quad \mathrm{N}(q) \neq 0 .
$$

Thus every non-zero real-quaternion has an inverse and $q^{-1}=\bar{q}$ for $\mathrm{N}(q)=1$. Further information about real-quaternions can be found in $[8,10,12]$.

Dual-numbers are an extension of real-numbers and are defined by introducing a new element $\varepsilon \neq 0$ (known as a dual unit) satisfying

$$
\varepsilon \neq 0, \quad 0 \varepsilon=\varepsilon 0=0, \quad r \varepsilon=\varepsilon r, \quad \varepsilon^{2}=0
$$

for all $r \in \mathbb{R}$. Thus, unlike real-quaternions, multiplication of dual-numbers is commutative. The set of dual-numbers is

$$
\mathbb{D}=\left\{A=a+\varepsilon a^{*} ; a, a^{*} \in \mathbb{R}\right\}
$$


where the real-numbers $a$ and $a^{*}$ are called, respectively, the scalar and dual parts of $A$. Dual-conjugate of $A$ is defined by $A^{*}=a-\varepsilon a^{*}$. The multiplication of dual-numbers $A=a+\varepsilon a^{*}$ and $B=b+\varepsilon b^{*}$ is given by the formula

$$
A B=(a b)+\varepsilon\left(a b^{*}+b a^{*}\right) .
$$

A dual-quaternion is a dual combination of two real-quaternions and thus the set of dual-quaternions is given as

$$
\mathbb{H}_{\mathbb{D}}=\left\{Q=q+\varepsilon q^{*} ; q, q^{*} \in \mathbb{H}\right\} .
$$

If $q$ and $q^{*}$ are pure, then $Q$ is said to be a pure and will be denoted by boldface letter $\boldsymbol{Q}$. A dual-quaternion $Q=q+\varepsilon q^{*}$ can also be written in the form

$$
Q=Q_{0}+Q_{1} \boldsymbol{i}+Q_{2} \boldsymbol{j}+Q_{3} \boldsymbol{k}
$$

where $Q_{i}=q_{i}+\varepsilon q_{i}^{*} \in \mathbb{D}, i=0,1,2,3 ; q=q_{0}+q_{1} \boldsymbol{i}+q_{2} \boldsymbol{j}+q_{3} \boldsymbol{k} \in \mathbb{H}, q^{*}=$ $q_{0}^{*}+q_{1}^{*} \boldsymbol{i}+q_{2}^{*} \boldsymbol{j}+q_{3}^{*} \boldsymbol{k} \in \mathbb{H}$. The basis vectors $\boldsymbol{i}, \boldsymbol{j}, \boldsymbol{k}$ satisfy the same multiplication rules with the basis vectors of real-quaternions given by Equation (1), and the product of $\varepsilon$ with $\boldsymbol{i}, \boldsymbol{j}, \boldsymbol{k}$ is commutative that is $\boldsymbol{i} \varepsilon=\varepsilon \boldsymbol{i}, \boldsymbol{j} \varepsilon=\varepsilon \boldsymbol{j}, \boldsymbol{k} \varepsilon=\varepsilon \boldsymbol{k}$.

The multiplication of dual-quaternions $Q=q+\varepsilon q^{*}$ and $P=p+\varepsilon p^{*}$ is

$$
Q P=(q p)+\varepsilon\left(q p^{*}+p q^{*}\right)=S_{Q} S_{P}-\left\langle\boldsymbol{V}_{Q}, \boldsymbol{V}_{P}\right\rangle+S_{Q} \boldsymbol{V}_{P}+S_{P} \boldsymbol{V}_{Q}+\boldsymbol{V}_{Q} \times \boldsymbol{V}_{P}
$$

where $S_{Q}=Q_{0}, S_{P}=P_{0}, \boldsymbol{V}_{Q}=Q_{1} \boldsymbol{i}+Q_{2} \boldsymbol{j}+Q_{3} \boldsymbol{k}, \boldsymbol{V}_{P}=P_{1} \boldsymbol{i}+P_{2} \boldsymbol{j}+P_{3} \boldsymbol{k}$. Also, $\left\langle\boldsymbol{V}_{Q}, \boldsymbol{V}_{P}\right\rangle=Q_{1} P_{1}+Q_{2} P_{2}+Q_{3} P_{3}$ and $\boldsymbol{V}_{Q} \times \boldsymbol{V}_{P}=\left(Q_{2} P_{3}-Q_{3} P_{2}\right) \boldsymbol{i}+$ $\left(Q_{3} P_{1}-Q_{1} P_{3}\right) \boldsymbol{j}+\left(Q_{1} P_{2}-Q_{2} P_{1}\right) \boldsymbol{k}$ denotes, respectively, the usual inner and vector products of $\boldsymbol{V}_{Q}$ and $\boldsymbol{V}_{P}$ in $\mathbb{D}^{3}$.

The algebra of dual-quaternions $\mathbb{H}_{\mathbb{D}}$ is isomorphic to the Clifford algebra $\mathrm{Cl}_{0,2,2}$ (i.e., $\mathbb{H}_{\mathbb{D}} \cong \mathrm{Cl}_{0,2,2}$ ) in dimension four where the generators $e_{i}, i=1, \ldots, 4$, satisfy

$$
e_{1}^{2}=e_{2}^{2}=-1, \quad e_{3}^{2}=e_{4}^{2}=0 \quad \text { and } \quad e_{i} e_{j}=-e_{j} e_{i} \quad \text { for } \quad i \neq j
$$

when the quaternionic units $\boldsymbol{i}, \boldsymbol{j}, \boldsymbol{k}$ are defined as, $e_{1}, e_{2}, e_{12}\left(=e_{1} e_{2}\right)$ while the dual unit $\varepsilon$ can be identified with $e_{34}\left(=e_{3} e_{4}\right)$, where $e_{34}$ commutes with a subalgebra $A$, which is isomorphic to $\mathbb{H}$, of $\mathrm{Cl}_{0,2,2}$ generated by $e_{1}, e_{2}$. Thus, multiplication in $\mathbb{H}_{\mathbb{D}}$ is associative.

The quaternionic-conjugation of a dual-quaternion $Q=q+\varepsilon q^{*}=Q_{0}+Q_{1} \boldsymbol{i}+$ $Q_{2} \boldsymbol{j}+Q_{3} \boldsymbol{k}$ is the standard involution $\bar{Q}=\bar{q}+\varepsilon \overline{q^{*}}=Q_{0}-Q_{1} \boldsymbol{i}-Q_{2} \boldsymbol{j}-Q_{3} \boldsymbol{k}$, which amounts to the canonical involution of $\mathbb{H}_{\mathbb{D}}$ as the Clifford algebra $\mathrm{Cl}_{0,2,2}$, and the norm of $Q$ is 


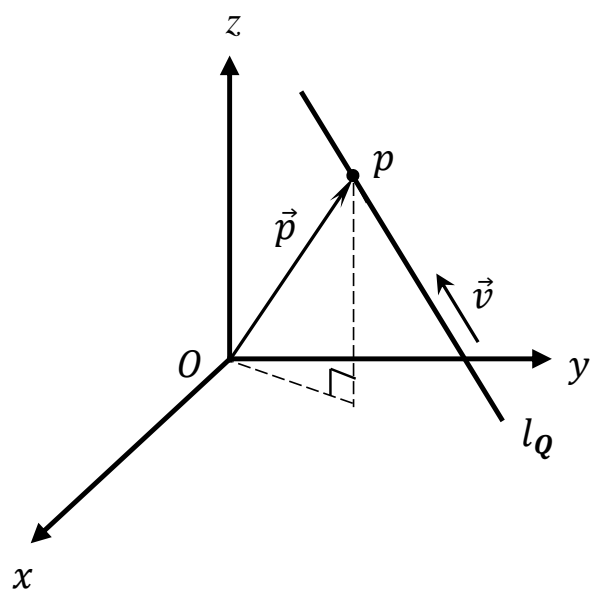

Figure 1. Geometry of the Plucker line in $\mathbb{R}^{3}$.

$$
\mathrm{N}(Q)=Q \bar{Q}=\bar{Q} Q=q \bar{q}+\varepsilon\left(q \bar{q}^{*}+q^{*} \bar{q}\right)=Q_{0}^{2}+Q_{1}^{2}+Q_{2}^{2}+Q_{3}^{2} \in \mathbb{D} .
$$

If $\mathrm{N}(Q)=1$, then $Q$ is said to be a unit dual-quaternion.

The multiplicative-inverse of $Q$ is

$$
Q^{-1}=\frac{\bar{Q}}{\mathrm{~N}(Q)}, \quad \mathrm{N}(Q) \neq 0 .
$$

That means, a non-zero dual-quaternion with a zero scalar part does not have an inverse and this differs dual-quaternions from real-quaternions, because every nonzero real-quaternion has an inverse. Also, $Q^{-1}=\bar{Q}$ for $\mathrm{N}(Q)=1$.

A non-zero dual-quaternion $Q=Q_{0}+Q_{1} \boldsymbol{i}+Q_{2} \boldsymbol{j}+Q_{3} \boldsymbol{k}$ can be represented in polar form as

$$
Q=\sqrt{\mathrm{N}(Q)}\left(\cos \frac{\phi}{2}+\vec{\eta} \sin \frac{\phi}{2}\right), \quad \phi \in \mathbb{D}
$$

where

$$
\begin{gathered}
\cos \frac{\phi}{2}=\frac{Q_{0}}{\sqrt{\mathrm{N}(Q)}}, \quad \sin \frac{\phi}{2}=\frac{\sqrt{Q_{1}^{2}+Q_{2}^{2}+Q_{3}^{2}}}{\sqrt{\mathrm{N}(Q})} \\
\vec{\eta}=\frac{Q_{1} \boldsymbol{i}+Q_{2} \boldsymbol{j}+Q_{3} \boldsymbol{k}}{\sqrt{Q_{1}^{2}+Q_{2}^{2}+Q_{3}^{2}}} \quad \text { for } \quad Q_{1}^{2}+Q_{2}^{2}+Q_{3}^{2} \neq 0 .
\end{gathered}
$$

According to E. Study there is a map, such that each point of the unit dual-sphere is in one-to-one correspondence with a line (known as the Plucker line) in $\mathbb{R}^{3}$. 
This correspondence can be given as follows. Let $\boldsymbol{Q}=\boldsymbol{q}+\varepsilon \boldsymbol{q}^{*}$ be a unit pure dual-quaternion. The scalar part $\boldsymbol{q}=\vec{v}$ is the direction vector of the line $l_{\boldsymbol{Q}}$ corresponding to $\boldsymbol{Q}$, and the dual part $\boldsymbol{q}^{*}=p \times \vec{v}$ is the moment of $\vec{v}$ about a chosen reference origin $O$ where $p$ is a point anywhere on the line $l_{\boldsymbol{Q}}$, see Fig. 1.

Example 1. The line $l_{\boldsymbol{P}}$ corresponding to unit pure dual-quaternion $\boldsymbol{P}=\boldsymbol{i}+z \boldsymbol{j} \varepsilon-$ $y \boldsymbol{k} \varepsilon$ has the direction vector $\vec{v}=(1,0,0) \in \mathbb{R}^{3}$ and passes through the point $P=(1, y, z) \in \mathbb{R}^{3}$, see Fig. 2.

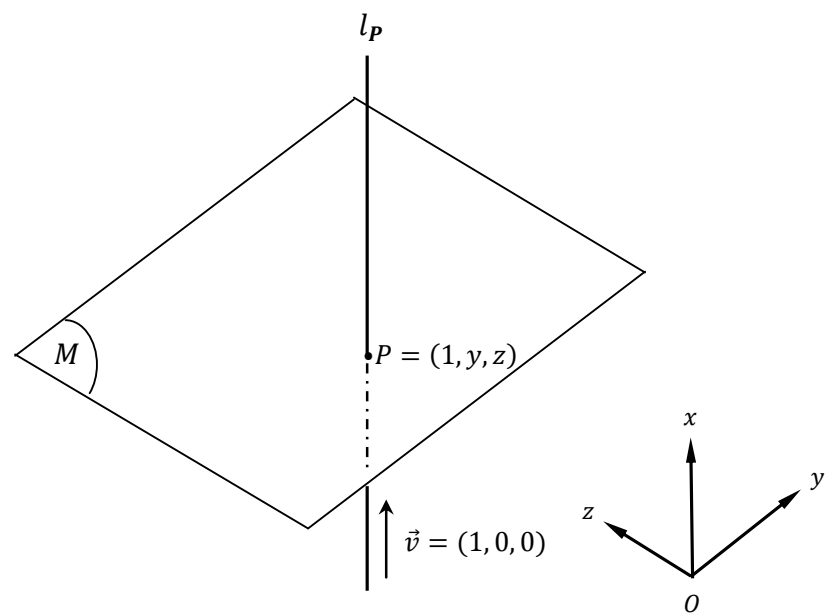

Figure 2. $l_{\boldsymbol{P}}$ is the line corresponding to unit pure dual-qaternion $\boldsymbol{P}$ and $M$ denotes the plane $x=1$ of $\mathbb{R}^{3}$.

Unit dual-quaternions are powerful tools of representing rigid-body (screw) motions in $\mathbb{R}^{3}$. A rotation of a line $l_{\boldsymbol{P}}$ (corresponding to unit pure dual-quaternion $\boldsymbol{P}$ ) about the unit axis vector $\overrightarrow{\boldsymbol{n}}=\left(n_{x}, n_{y}, n_{z}\right)$ by an angle $\theta \in \mathbb{R}$ can be represented by the unit dual-quaternion

$$
Q_{r}=\cos \left(\frac{\theta}{2}\right)+n_{x} \sin \left(\frac{\theta}{2}\right) \boldsymbol{i}+n_{y} \sin \left(\frac{\theta}{2}\right) \boldsymbol{j}+n_{z} \sin \left(\frac{\theta}{2}\right) \boldsymbol{k}+0 \varepsilon
$$

as

$$
Q_{r} \boldsymbol{P} Q_{r}^{-1}=Q_{r} \boldsymbol{P} \bar{Q}_{r} .
$$

A translation of the line $l_{\boldsymbol{P}}$ by a magnitude $t=\left(t_{1}, t_{2}, t_{3}\right) \in \mathbb{R}^{3}$ along $\overrightarrow{\boldsymbol{n}}=$ $\left(n_{x}, n_{y}, n_{z}\right)$ can also be represented by the dual-quaternion

$$
Q_{t}=1+\frac{\varepsilon}{2}\left(0+t_{1} \boldsymbol{i}+t_{2} \boldsymbol{j}+t_{3} \boldsymbol{k}\right)
$$


as

$$
Q_{t} \boldsymbol{P} Q_{t}^{-1}=Q_{t} \boldsymbol{P} \bar{Q}_{t} .
$$

Thus, a rotation (by an angle $\theta \in \mathbb{R}$ ) followed by a translation (by magnitude $t \in \mathbb{R}^{3}$ ) can be represented by the dual-quaternion $Q=Q_{t} Q_{r}$ as

$$
Q \boldsymbol{P} Q^{-1}=Q \boldsymbol{P} \bar{Q}
$$

where the axis of this screw-motion is the unit vector $\overrightarrow{\boldsymbol{n}}=\left(n_{x}, n_{y}, n_{z}\right)$. For further information about dual-quaternions see $[1,3,6]$.

The set of semi-quaternions can be given as

$$
\mathbb{H}_{s}=\left\{q=q_{0}+q_{1} \mathbf{i}+q_{2} \mathbf{j}+q_{3} \mathbf{k} ; q_{0}, q_{1}, q_{2}, q_{3} \in \mathbb{R}\right\}
$$

where the basis elements $\mathbf{i}, \mathbf{j}, \mathbf{k}$ (to distinguish the basis elements of semi - quaternions from the basis elements of real- and dual-quaternions, we have used upright letters for the basis elements of semi-quaternions) satisfy the non-commutative multiplication rules

$$
\mathbf{i}^{2}=-1, \quad \mathbf{j}^{2}=\mathbf{k}^{2}=0, \quad \mathbf{i j}=-\mathbf{j i}=\mathbf{k}, \quad \mathbf{j k}=-\mathbf{k} \mathbf{j}=0, \quad \mathbf{k i}=-\mathbf{i k}=\mathbf{j} .
$$

Here again $S_{q}=q_{0}$ and $\boldsymbol{V}_{q}=q_{1} \mathbf{i}+q_{2} \mathbf{j}+q_{3} \mathbf{k}$ are called, respectively, the scalar and the vector parts of $q=q_{0}+q_{1} \mathbf{i}+q_{2} \mathbf{j}+q_{3} \mathbf{k}$. If $S_{q}=0$, then $q=\boldsymbol{V}_{q}$ is said to be a pure and will be denoted by boldface letter $\boldsymbol{q}$. The set of all pure semi-quaternions will be denoted by $\hat{\mathbb{H}}_{s}$.

The multiplication of two semi-quaternions $q=S_{q}+\boldsymbol{V}_{q}$ and $p=S_{p}+\boldsymbol{V}_{p}$ is

$$
\begin{aligned}
q p= & S_{q} S_{p}-\left\langle\boldsymbol{V}_{q}, \boldsymbol{V}_{p}\right\rangle_{s}+S_{q} \boldsymbol{V}_{p}+S_{p} \boldsymbol{V}_{q}+\boldsymbol{V}_{q} \times_{s} \boldsymbol{V}_{p} \\
= & \left(q_{0} p_{0}-q_{1} p_{1}\right)+\left(q_{1} p_{0}+q_{0} p_{1}\right) \mathbf{i}+\left(q_{2} p_{0}+q_{3} p_{1}+q_{0} p_{2}-q_{1} p_{3}\right) \mathbf{j} \\
& +\left(q_{3} p_{0}-q_{2} p_{1}+q_{1} p_{2}-q_{0} p_{3}\right) \mathbf{k}
\end{aligned}
$$

where $S_{q}=q_{0}, S_{p}=p_{0}, \boldsymbol{V}_{q}=q_{1} \mathbf{i}+q_{2} \mathbf{j}+q_{3} \mathbf{k}, \boldsymbol{V}_{p}=p_{1} \mathbf{i}+p_{2} \mathbf{j}+p_{3} \mathbf{k}$ and $\left\langle\boldsymbol{V}_{q}, \boldsymbol{V}_{p}\right\rangle_{s}$ $=q_{1} p_{1}, \boldsymbol{V}_{q} \times{ }_{s} \boldsymbol{V}_{p}=0 \mathbf{i}+\left(q_{3} p_{1}-q_{1} p_{3}\right) \mathbf{j}+\left(q_{1} p_{2}-q_{2} p_{1}\right) \mathbf{k}$.

The algebra of semi-quaternions $\mathbb{H}_{\mathrm{s}}$ is isomorphic to the Clifford algebra $\mathrm{Cl}_{0,1,1}$ (i.e., $\mathbb{H}_{\mathrm{s}} \cong \mathrm{Cl}_{0,1,1}$ ) in dimension two where the standard anti-commuting generators $e_{1}, e_{2}$ satisfy

$$
e_{1}^{2}=-1, \quad e_{2}^{2}=\left(e_{1} e_{2}\right)^{2}=0 \quad \text { and } \quad e_{1} e_{2}=-e_{2} e_{1}
$$

when the semi-quaternionic units $\mathbf{i}, \mathbf{j}, \mathbf{k}$ are defined with $e_{1}, e_{2}, e_{12}\left(=e_{1} e_{2}\right) \in$ $\mathrm{Cl}_{0,1,1}$ respectively. Hence the multiplication in $\mathbb{H}_{\mathrm{s}}$ is associative. 
The quaternionic-conjugation of the semi-quaternion $q=q_{0}+q_{1} \mathbf{i}+q_{2} \mathbf{j}+q_{3} \mathbf{k}$ is the standard involution $\bar{q}=q_{0}-q_{1} \mathbf{i}-q_{2} \mathbf{j}-q_{3} \mathbf{k}$, which amounts to the canonical involution of $\mathbb{H}_{s}$ as the Clifford algebra $\mathrm{Cl}_{0,1,1}$, and the norm of $q=q_{0}+q_{1} \mathbf{i}+$ $q_{2} \mathbf{j}+q_{3} \mathbf{k}$ is

$$
\mathrm{N}(q)=q \bar{q}=\bar{q} q=q_{0}^{2}+q_{1}^{2} \in \mathbb{R} .
$$

If $\mathrm{N}(q)=1$, then $q$ is said to be a unit semi-quaternion. The set of all unit semiquaternions will be denoted by $\mathbb{H}_{s 1}$ while the set of all unit pure semi-quaternions will be denoted by $\hat{\mathbb{H}}_{s 1}$.

For arbitrary semi-quaternions $q, p, r$ the following properties are valid

$$
\overline{\bar{q}}=q, \quad \overline{q+p}=\bar{q}+\bar{p}=\bar{p}+\bar{q}, \quad \overline{q p r}=\overline{r p q} .
$$

The multiplicative-inverse of $q$ is

$$
q^{-1}=\frac{\bar{q}}{\mathrm{~N}(q)}
$$

for $q_{0} \neq 0 \neq q_{1}$. That means a non-zero semi-quaternion $q=q_{0}+q_{1} \mathbf{i}+q_{2} \mathbf{j}+q_{3} \mathbf{k}$ with $q_{0}=0=q_{1}$ does not have an inverse. This case differs semi-quaternions from real-quaternions, because a non-zero real-quaternion has an inverse.

A semi-quaternion $q=q_{0}+q_{1} \mathbf{i}+q_{2} \mathbf{j}+q_{3} \mathbf{k}$ can be expressed in complex form as

$$
q=a+b \vec{\mu}
$$

where

$$
a=q_{0}, \quad b=\sqrt{q_{1}^{2}}, \quad \vec{\mu}=\frac{q_{1} \mathbf{i}+q_{2} \mathbf{j}+q_{3} \mathbf{k}}{b} \text { for } \quad b \neq 0 .
$$

Also, a non-zero semi-quaternion $q=q_{0}+q_{1} \mathbf{i}+q_{2} \mathbf{j}+q_{3} \mathbf{k}$ can be represented in polar form as

$$
q=\sqrt{\mathrm{N}(q)}\left(\cos \frac{\theta}{2}+\vec{w} \sin \frac{\theta}{2}\right), \quad \theta \in \mathbb{R}
$$

where

$$
\begin{gathered}
\cos \frac{\theta}{2}=\frac{\left|q_{0}\right|}{\sqrt{\mathrm{N}(q)}}, \quad \sin \frac{\theta}{2}=\frac{\left|q_{1}\right|}{\sqrt{\mathrm{N}(q)}} \\
\vec{w}=\frac{1}{\left|q_{1}\right|}\left(q_{1} \mathbf{i}+q_{2} \mathbf{j}+q_{3} \mathbf{k}\right) \text { for } q_{1} \neq 0 .
\end{gathered}
$$

For further information about semi-quaternions see $[9,11]$. 


\section{Semi-Quaternion Involutions and Anti-Involutions}

In this section, we will represent an involution and an anti-involution mapping obtained by semi-quaternions, and a geometric interpretation of each as planarmotions in $\mathbb{R}^{3}$.

Theorem 2. Let $q$ be an arbitrary semi-quaternion. Then the mapping

$$
f_{v}(q)=-v \bar{q} v
$$

is an anti-involution for a chosen unit pure semi-quaternion $\boldsymbol{v}$.

Proof: Self-inverse axiom can be shown to be satisfied by the mapping $f_{v}$ as

$$
f_{\boldsymbol{v}}\left(f_{\boldsymbol{v}}(q)\right)=f_{\boldsymbol{v}}(-\boldsymbol{v} \bar{q} \boldsymbol{v})=-\boldsymbol{v}(-\overline{\boldsymbol{v}} \bar{q} \boldsymbol{v}) \boldsymbol{v}=-\boldsymbol{v}(-\overline{\boldsymbol{v}} \overline{\bar{q}} \overline{\boldsymbol{v}}) \boldsymbol{v}=-\boldsymbol{v}((-\overline{\boldsymbol{v}}) q \overline{\boldsymbol{v}}) \boldsymbol{v}=\boldsymbol{v}^{2} q \boldsymbol{v}^{2}
$$

and since $v$ is unit pure semi-quaternion, it is $v^{2}=-1$, thus

$$
f_{v}\left(f_{v}(q)\right)=q .
$$

The linearity axiom can also be shown to be satisfied as

$$
f_{\boldsymbol{v}}(\lambda q)=-\boldsymbol{v}(\overline{\lambda q}) \boldsymbol{v}=\lambda(-\boldsymbol{v} \bar{q} \boldsymbol{v})=\lambda f_{\boldsymbol{v}}(q)
$$

and

$$
f_{\boldsymbol{v}}(q+p)=-\boldsymbol{v}(\overline{q+p}) \boldsymbol{v}=f_{\boldsymbol{v}}(q)+f_{\boldsymbol{v}}(p)
$$

where $p \in \mathbb{H}_{s}$ and $\lambda \in \mathbb{R}$. Finally, the anti-homomorphism axiom can be shown to be satisfied as

$$
f_{\boldsymbol{v}}(q p)=-\boldsymbol{v}(\overline{q p}) \boldsymbol{v}=-\boldsymbol{v}(\bar{p} \bar{q}) \boldsymbol{v}
$$

and since $\boldsymbol{v}$ is unit pure, it is $-\boldsymbol{v} \boldsymbol{v}=-\boldsymbol{v}^{2}=1$, thus

$$
f_{\boldsymbol{v}}(q p)=-\boldsymbol{v} \bar{p}(-\boldsymbol{v} \boldsymbol{v}) \bar{q} \boldsymbol{v}=(-\boldsymbol{v} \bar{p} \boldsymbol{v})(-\boldsymbol{v} \bar{q} \boldsymbol{v})=f_{\boldsymbol{v}}(p) f_{\boldsymbol{v}}(q) .
$$

Theorem 3. Let $q$ be an arbitrary semi-quaternion. Then the mapping

$$
f_{v}(q)=-v q v
$$

is an involution for a chosen unit pure semi-quaternion $\boldsymbol{v}$. 
Proof: Let $q$ be a semi-quaternion, then

$$
f_{\boldsymbol{v}}\left(f_{\boldsymbol{v}}(q)\right)=f_{\boldsymbol{v}}(-\boldsymbol{v} q \boldsymbol{v})=-\boldsymbol{v}(-\boldsymbol{v} q \boldsymbol{v}) \boldsymbol{v}=\boldsymbol{v}^{2} q \boldsymbol{v}^{2}=q
$$

thus $f_{v}$ is self-inverse. Furthermore

$$
f_{\boldsymbol{v}}(\lambda q)=-\boldsymbol{v}(\lambda q) \boldsymbol{v}=\lambda(-\boldsymbol{v} q \boldsymbol{v})=\lambda f_{\boldsymbol{v}}(q)
$$

and

$$
f_{\boldsymbol{v}}(q+p)=-\boldsymbol{v}(q+p) \mathbf{v}=f_{\boldsymbol{v}}(q)+f_{\boldsymbol{v}}(p)
$$

where $p \in \mathbb{H}_{s}$ and $\lambda \in \mathbb{R}$ that means $f_{v}$ is linear. The homomorphism axiom can be shown to be satisfied as

$$
f_{\boldsymbol{v}}(q p)=-\boldsymbol{v}(q p) \boldsymbol{v}=-\boldsymbol{v} q(-\boldsymbol{v} \boldsymbol{v}) p \boldsymbol{v}=(-\boldsymbol{v} q \boldsymbol{v})(-\boldsymbol{v} p \boldsymbol{v})=f_{\boldsymbol{v}}(q) f_{\boldsymbol{v}}(p) .
$$

\subsection{Geometry of the Semi-Quaternion (Anti)-Involutions}

A planar-rotation in three-dimensional Euclidean space $\mathbb{R}^{3}$ can be represented by a unit semi-quaternion

$q=\cos \left(\frac{\theta}{2}\right)+\sin \left(\frac{\theta}{2}\right) \mathbf{i}+\left(\frac{t_{2}}{2} \cos \left(\frac{\theta}{2}\right)+\frac{t_{3}}{2} \sin \left(\frac{\theta}{2}\right)\right) \mathbf{j}+\left(-\frac{t_{2}}{2} \sin \left(\frac{\theta}{2}\right)+\frac{t_{3}}{2} \cos \left(\frac{\theta}{2}\right)\right) \mathbf{k}$ as follows. Let $\boldsymbol{p}=\mathbf{i}+z \mathbf{j}-y \mathbf{k} \in \hat{\mathbb{H}}_{s 1}$, then

$$
\boldsymbol{p}^{\prime}=q \boldsymbol{p} q^{-1}=\mathbf{i}+\left(\cos \theta z+\sin \theta y+t_{3}\right) \mathbf{j}-\left(\cos \theta y-\sin \theta z+t_{2}\right) \mathbf{k} \in \hat{\mathbb{H}}_{s 1} .
$$

If we associate with an arbitrary semi-quaternion $r=r_{0}+r_{1} \mathbf{i}+r_{2} \mathbf{j}+r_{3} \mathbf{k}$ the dual-quaternion $R=\left(r_{0}+r_{1} \boldsymbol{i}\right)+\varepsilon\left(r_{2} \boldsymbol{j}+r_{3} \boldsymbol{k}\right)$ that is

$$
r=r_{0}+r_{1} \mathbf{i}+r_{2} \mathbf{j}+r_{3} \mathbf{k} \in \mathbb{H}_{s} \triangleq R=\left(r_{0}+r_{1} \boldsymbol{i}\right)+\varepsilon\left(r_{2} \boldsymbol{j}+r_{3} \boldsymbol{k}\right) \in \mathbb{H}_{\mathbb{D}}
$$

then the unit pure semi-quaternions $\boldsymbol{p}, \boldsymbol{p}^{\prime}$ can be given respectively by the dualquaternions

$$
\begin{gathered}
\boldsymbol{P}=\boldsymbol{i}+z \mathbf{j} \varepsilon-y \boldsymbol{k} \varepsilon \\
\boldsymbol{P}^{\prime}=\boldsymbol{i}+\left(\cos \theta z+\sin \theta \mathbf{y}+t_{3}\right) \boldsymbol{j} \varepsilon-\left(\cos \theta y-\sin \theta z+t_{2}\right) \boldsymbol{k} \varepsilon .
\end{gathered}
$$

Thus, $\boldsymbol{P}$ and $\boldsymbol{P}^{\prime}$ are planar. The line $l_{\boldsymbol{P}}$ corresponding to the unit pure dual-quaternion $\boldsymbol{P}$ has the direction vector $\vec{v}=(1,0,0) \in \mathbb{R}^{3}$ and intersects the plane $x=1$ of $\mathbb{R}^{3}$ at the point $P=(1, y, z) \in \mathbb{R}^{3}$, while the line $l_{\boldsymbol{P}^{\prime}}$ corresponding to the unit 
pure dual-quaternion $\boldsymbol{P}^{\prime}$ has also the same direction vector with $l_{\boldsymbol{P}}$ and intersects the plane $x=1$ of $\mathbb{R}^{3}$ at the point

$$
P^{\prime}=\left(1, \cos \theta y-\sin \theta z+t_{2}, \cos \theta z+\sin \theta y+t_{3}\right) \in \mathbb{R}^{3}
$$

so that $P$ and $P^{\prime}$ are also planar. Let $M$ be the plane $x=1$ of $\mathbb{R}^{3}$, then the map

$$
f_{q}: M \rightarrow M
$$

defined by

$$
P=(1, y, z) \mapsto P^{\prime}=\left(1, \cos \theta y-\sin \theta z+t_{2}, \cos \theta z+\sin \theta y+t_{3}\right)
$$

is linear for a chosen unit semi-quaternion

$$
q=\cos \left(\frac{\theta}{2}\right)+\sin \left(\frac{\theta}{2}\right) \mathbf{i}+\left(\frac{t_{2}}{2} \cos \left(\frac{\theta}{2}\right)+\frac{t_{3}}{2} \sin \left(\frac{\theta}{2}\right)\right) \mathbf{j}+\left(-\frac{t_{2}}{2} \sin \left(\frac{\theta}{2}\right)+\frac{t_{3}}{2} \cos \left(\frac{\theta}{2}\right)\right) \mathbf{k} .
$$

The matrix representation of the map $f_{q}$ can be given by

$$
N=\left(\begin{array}{ccc}
1 & 0 & 0 \\
t_{2} & \cos \theta & -\sin \theta \\
t_{3} & \sin \theta & \cos \theta
\end{array}\right)
$$

and it can be easily checked that $N^{t} \varepsilon N=\varepsilon$ and $\operatorname{det} N=1$ for $\varepsilon=\operatorname{diag}(1,0,0)$ thus $N$ is orthogonal so that the linear map $f_{q}(P)=P^{\prime}$ represents a rotation.

Proposition 4. A unit semi-quaternion $q=q_{0}+q_{1} \mathbf{i}+q_{2} \mathbf{j}+q_{3} \mathbf{k}$ defined by

$$
\begin{aligned}
q_{0} & =\cos \left(\frac{\theta}{2}\right), & q_{1} & =\sin \left(\frac{\theta}{2}\right) \\
q_{2} & =\frac{t_{2}}{2} \cos \left(\frac{\theta}{2}\right)+\frac{t_{3}}{2} \sin \left(\frac{\theta}{2}\right), & q_{3} & =-\frac{t_{2}}{2} \sin \left(\frac{\theta}{2}\right)+\frac{t_{3}}{2} \cos \left(\frac{\theta}{2}\right)
\end{aligned}
$$

represents a positive oriented rotation in two-dimensional Euclidean space $\mathbb{R}^{2}$ by an angle $\theta \in \mathbb{R}$ and center

$$
m=\left(-\frac{q_{3}}{\sin \left(\frac{\theta}{2}\right)}, \frac{q_{2}}{\sin \left(\frac{\theta}{2}\right)}\right) \in \mathbb{R}^{2} .
$$

Proof: A planar-motion in the plane $\mathbb{R}^{2}$ can be given by the map

$$
\beta_{q}: \mathbb{R}^{2} \rightarrow \mathbb{R}^{2}, \quad\left(\begin{array}{l}
x \\
y
\end{array}\right) \mapsto\left(\begin{array}{cc}
\cos \theta & -\sin \theta \\
\sin \theta & \cos \theta
\end{array}\right)\left(\begin{array}{l}
x \\
y
\end{array}\right)+\left(\begin{array}{c}
t_{2} \\
t_{3}
\end{array}\right)
$$

where

$$
\cos \theta=q_{0}^{2}-q_{1}^{2}, \quad \sin \theta=-2 q_{0} q_{1}
$$




$$
t_{2}=2\left(q_{1} q_{2}+q_{0} q_{3}\right), \quad t_{2}=2\left(q_{0} q_{2}-q_{1} q_{3}\right) .
$$

It is straightforward to see that

$$
\beta_{q}\left(-\frac{q_{3}}{\sin \left(\frac{\theta}{2}\right)}, \frac{q_{2}}{\sin \left(\frac{\theta}{2}\right)}\right)=\left(-\frac{q_{3}}{\sin \left(\frac{\theta}{2}\right)}, \frac{q_{2}}{\sin \left(\frac{\theta}{2}\right)}\right)
$$

and thus the linear map $\beta_{q}$ represents a positive oriented rotation with an angle $\theta \in \mathbb{R}$ and center

$$
m=\left(-\frac{q_{3}}{\sin \left(\frac{\theta}{2}\right)}, \frac{q_{2}}{\sin \left(\frac{\theta}{2}\right)}\right) \in \mathbb{R}^{2}
$$

in two-dimensional Euclidean space $\mathbb{R}^{2}$.

Corollary 5. Let $\boldsymbol{q}=\mathbf{i}+q_{2} \mathbf{j}+q_{3} \mathbf{k}$ be a unit pure semi-quaternion. Then, the product $\boldsymbol{q} \boldsymbol{p} \boldsymbol{q}^{-1}=-\boldsymbol{q p} \boldsymbol{q}$ represents a planar-reflection under the following two conditions

1. A reflection of the point $P_{1}=(1, y, z)$ in the plane $x=1$ of $\mathbb{R}^{3}$ through the point $\mathcal{P}_{1}=\left(1,-q_{3}, q_{2}\right)$ if $\boldsymbol{p}=\mathbf{i}+z \mathbf{j}-y \mathbf{k} \in \hat{\mathbb{H}}_{s 1}$.

2. A reflection of the point $P_{2}=(-1, y, z)$ in the plane $x=-1$ of $\mathbb{R}^{3}$ through the point $\mathcal{P}_{2}=\left(-1, q_{3},-q_{2}\right)$ if $\boldsymbol{p}=-\mathbf{i}+z \mathbf{j}-y \mathbf{k} \in \hat{\mathbb{H}}_{s 1}$.

Proof: If we take $\cos (\theta / 2)=0$ and $\sin (\theta / 2)=1$ in equation (11), we obtain

$$
\boldsymbol{q}=\mathbf{i}+\left(\frac{t_{3}}{2}\right) \mathbf{j}-\left(\frac{t_{2}}{2}\right) \mathbf{k}, \quad t_{3} / 2=q_{2}, t_{2} / 2=-q_{3}
$$

Thus

1. If $\boldsymbol{p}=\mathbf{i}+z \mathbf{j}-y \mathbf{k} \in \hat{\mathbb{H}}_{s 1}$, then the map $f_{\mathbf{q}}=\boldsymbol{q} \boldsymbol{p} \boldsymbol{q}^{-1}=-\boldsymbol{q} \boldsymbol{p} \boldsymbol{q}$ given by the Equation 10 becomes

$$
P_{1}=(1, y, z) \in M \mapsto P_{1}^{\prime}=\left(1,-y+t_{2},-z+t_{3}\right) \in M
$$

so that the product $f_{q}$ represents a reflection of the point $P_{1}=(1, y, z)$ in the plane $x=1$ of $\mathbb{R}^{3}$ through the point $\mathcal{P}_{1}=\left(1, t_{2} / 2, t_{3} / 2\right)$.

2. If $\boldsymbol{p}=-\mathbf{i}+z \mathbf{j}-y \mathbf{k} \in \hat{\mathbb{H}}_{s 1}$, then the product $\boldsymbol{q} \boldsymbol{p} \boldsymbol{q}^{-1}=-\boldsymbol{q} \boldsymbol{p} \boldsymbol{q}$ results to

$$
P_{2}=(-1, y, z) \in \mathbb{R}^{3} \mapsto P_{2}^{\prime}=\left(-1,-y-t_{2},-z-t_{3}\right) \in \mathbb{R}^{3}
$$


so that the product $\boldsymbol{q} \boldsymbol{p} \boldsymbol{q}^{-1}=-\boldsymbol{q p} \boldsymbol{q}$ represents a reflection of the point $P_{2}=$ $(-1, y, z)$ in the plane $x=-1$ of $\mathbb{R}^{3}$ through the point $\mathcal{P}_{2}=-\left(1, t_{2} / 2, t_{3} / 2\right)$.

Corollary 6. Let $\boldsymbol{q}=-\mathbf{i}+q_{2} \mathbf{j}+q_{3} \mathbf{k}$ be a unit pure semi-quaternion. Then, the product $\boldsymbol{q p q}^{-1}=-\boldsymbol{q p q}$ represents a planar-reflection under the following two conditions

1. A reflection of the point $P_{1}=(1, y, z)$ in the plane $x=1$ of $\mathbb{R}^{3}$ through the point $\mathcal{Q}_{1}=\left(1, q_{3}, q_{2}\right)$ if $\boldsymbol{p}=\mathbf{i}+z \mathbf{j}-y \mathbf{k} \in \hat{\mathbb{H}}_{s 1}$.

2. A reflection of the point $P_{2}=(-1, y, z)$ in the plane $x=-1$ of $\mathbb{R}^{3}$ through the point $\mathcal{Q}_{2}=-\left(1, q_{3}, q_{2}\right)$ if $\boldsymbol{p}=-\mathbf{i}+z \mathbf{j}-y \mathbf{k} \in \hat{\mathbb{H}}_{s 1}$.

The proof of Corollary 6 is similar to the proof of Corollary 5 .

Proposition 7. For an arbitrary semi-quaternion $q=a+b \vec{\mu}$, the involution map $f_{v}(q)=-\boldsymbol{v} q \boldsymbol{v}$ given by Theorem 3, leaves the scalar part " $a$ ” of $q$ invariant and

1. reflects the point $K_{1}=\left(1,-\mu_{3}, \mu_{2}\right) b$ in the plane $x=1$ of $\mathbb{R}^{3}$ through the point $\mathcal{K}_{1}=\left(1,-v_{3}, v_{2}\right)$ if $\boldsymbol{v}=\mathbf{i}+v_{2} \mathbf{j}+v_{3} \mathbf{k} \in \hat{\mathbb{H}}_{s 1}, \vec{\mu}=\mathbf{i}+\mu_{2} \mathbf{j}+\mu_{3} \mathbf{k} \in \hat{\mathbb{H}}_{s 1}$.

2. reflects the point $K_{2}=\left(-1,-\mu_{3}, \mu_{2}\right) b$ in the plane $x=-1$ of $\mathbb{R}^{3}$ through the point $\mathcal{K}_{2}=\left(-1, v_{3},-v_{2}\right)$ if $\boldsymbol{v}=\mathbf{i}+v_{2} \mathbf{j}+v_{3} \mathbf{k} \in \hat{\mathbb{H}}_{s 1}, \vec{\mu}=-\mathbf{i}+\mu_{2} \mathbf{j}+$ $\mu_{3} \mathbf{k} \in \hat{\mathbb{H}}_{s 1}$.

3. reflects the point $K_{3}=\left(1,-\mu_{3}, \mu_{2}\right) b$ in the plane $x=1$ of $\mathbb{R}^{3}$ through the point $\mathcal{K}_{3}=\left(1, v_{3}, v_{2}\right)$ if $\boldsymbol{v}=-\mathbf{i}+v_{2} \mathbf{j}+v_{3} \mathbf{k} \in \hat{\mathbb{H}}_{s 1}, \vec{\mu}=\mathbf{i}+\mu_{2} \mathbf{j}+\mu_{3} \mathbf{k} \in \hat{\mathbb{H}}_{s 1}$.

4. reflects the point $K_{4}=\left(-1,-\mu_{3}, \mu_{2}\right) b$ in the plane $x=-1$ of $\mathbb{R}^{3}$ through the point $\mathcal{K}_{4}=-\left(1, v_{3}, v_{2}\right)$ if $\boldsymbol{v}=-\mathbf{i}+v_{2} \mathbf{j}+v_{3} \mathbf{k} \in \hat{\mathbb{H}}_{s 1}, \vec{\mu}=-\mathbf{i}+\mu_{2} \mathbf{j}+$ $\mu_{3} \mathbf{k} \in \hat{\mathbb{H}}_{s 1}$.

Proof: Let $q=a+b \vec{\mu}$ be a semi-quaternion, then

$$
f_{\boldsymbol{v}}(q)=-\boldsymbol{v} q \boldsymbol{v}=-\boldsymbol{v}(a+b \vec{\mu}) \boldsymbol{v}=-\boldsymbol{v}^{2} a-\boldsymbol{v} \vec{\mu} \boldsymbol{v} b=a-\boldsymbol{v} \vec{\mu} \boldsymbol{v} b .
$$

Thus, the involution map $f_{\boldsymbol{v}}(q)=-\boldsymbol{v} q \boldsymbol{v}$ leaves the scalar part " $a$ " of $q$ invariant and

1. since $\boldsymbol{v}=\mathbf{i}+v_{2} \mathbf{j}+v_{3} \mathbf{k} \in \hat{\mathbb{H}}_{s 1}, \vec{\mu}=\mathbf{i}+\mu_{2} \mathbf{j}+\mu_{3} \mathbf{k} \in \hat{\mathbb{H}}_{s 1}$, thus from the first item in Corollary 5 , the product $-\boldsymbol{v} \vec{\mu} \boldsymbol{v}$ reflects the point $\left(1,-\mu_{3}, \mu_{2}\right)$ in the plane $x=1$ of $\mathbb{R}^{3}$ through the point $\left(1,-v_{3}, v_{2}\right)$. 
Also, the proofs of items 2, 3 and 4 in Proposition 7 are obvious from, respectively, the item 2 in Corollary 5 and items 1 and 2 in Corollary 6.

Proposition 8. For an arbitrary semi-quaternion $q=a+b \vec{\mu}$, the anti-involution map $f_{v}(q)=-\boldsymbol{v} \bar{q} \boldsymbol{v}$ given by Theorem 2, leaves the scalar part " $a$ " of $q$ invariant and

1. reflects the point $K_{1}=\left(1,-\mu_{3}, \mu_{2}\right) b$ through the point $\mathcal{L}_{1}=\left(0,-\mu_{3}+\right.$ $\left.v_{3}, \mu_{2}-v_{2}\right)$ in $\mathbb{R}^{3}$ if $\boldsymbol{v}=\mathbf{i}+v_{2} \mathbf{j}+v_{3} \mathbf{k} \in \hat{\mathbb{H}}_{s 1}, \vec{\mu}=\mathbf{i}+\mu_{2} \mathbf{j}+\mu_{3} \mathbf{k} \in \hat{\mathbb{H}}_{s 1}$.

2. reflects the point $K_{2}=\left(-1,-\mu_{3}, \mu_{2}\right) b$ through the point $\mathcal{L}_{2}=\left(0,-\mu_{3}-\right.$ $\left.v_{3}, \mu_{2}+v_{2}\right)$ in $\mathbb{R}^{3}$ if $\boldsymbol{v}=\mathbf{i}+v_{2} \mathbf{j}+v_{3} \mathbf{k} \in \hat{\mathbb{H}}_{s 1}, \vec{\mu}=-\mathbf{i}+\mu_{2} \mathbf{j}+\mu_{3} \mathbf{k} \in \hat{\mathbb{H}}_{s 1}$.

3. reflects the point $K_{3}=\left(1,-\mu_{3}, \mu_{2}\right) b$ through the point $\mathcal{L}_{3}=\left(0,-\mu_{3}-\right.$ $\left.v_{3}, \mu_{2}-v_{2}\right)$ in $\mathbb{R}^{3}$ if $\boldsymbol{v}=-\mathbf{i}+v_{2} \mathbf{j}+v_{3} \mathbf{k} \in \hat{\mathbb{H}}_{s 1}, \vec{\mu}=\mathbf{i}+\mu_{2} \mathbf{j}+\mu_{3} \mathbf{k} \in \hat{\mathbb{H}}_{s 1}$.

4. reflects the point $K_{4}=\left(-1,-\mu_{3}, \mu_{2}\right) b$ through the point $\mathcal{L}_{4}=\left(0,-\mu_{3}+\right.$ $\left.v_{3}, \mu_{2}+v_{2}\right)$ in $\mathbb{R}^{3}$ if $\boldsymbol{v}=-\mathbf{i}+v_{2} \mathbf{j}+v_{3} \mathbf{k} \in \hat{\mathbb{H}}_{s 1}, \vec{\mu}=-\mathbf{i}+\mu_{2} \mathbf{j}+\mu_{3} \mathbf{k} \in \hat{\mathbb{H}}_{s 1}$.

Proof: Let $q=a+b \vec{\mu}$ be a semi-quaternion, then

$$
f_{\boldsymbol{v}}(q)=-\boldsymbol{v} \bar{q} \boldsymbol{v}=-\boldsymbol{v}(\overline{a+b \vec{\mu}}) \boldsymbol{v}=-\boldsymbol{v}(a-b \vec{\mu}) \boldsymbol{v}=a+\boldsymbol{v} \vec{\mu} \boldsymbol{v} b .
$$

Thus, the anti-involution map $f_{\boldsymbol{v}}(q)=-\boldsymbol{v} q \boldsymbol{v}$ leaves the scalar part " $a$ " of $q$ invariant and

1. from the proof of item 1 in Corollary 5, the product $v \vec{\mu} v$ results to

$$
K_{1}=\left(1,-\mu_{3}, \mu_{2}\right) \in \mathbb{R}^{3} \mapsto K_{1}{ }^{\prime}=\left(-1,-\mu_{3}+2 v_{3}, \mu_{2}-2 v_{2}\right) \in \mathbb{R}^{3}
$$

which completes the proof.

The proofs of items 2, 3 and 4 in Proposition 8 can be easily checked by using, respectively, item 2 in Corollary 5 and items 1 and 2 in Corollary 6.

Example 9. Let

$$
\boldsymbol{v}=\mathbf{i}-\frac{1}{2} \mathbf{j}-\frac{1}{2} \mathbf{k} \in \hat{\mathbb{H}}_{s 1}, \quad q=1+\mathbf{i}+\mathbf{j}-\mathbf{k} \in \mathbb{H}_{s 1}
$$

then the product $-\boldsymbol{v} q \boldsymbol{v}($ which is an involution) becomes

$$
q^{\prime}=-\boldsymbol{v} q \boldsymbol{v}=1+\mathbf{i}-2 \mathbf{j} \in \mathbb{H}_{s 1}
$$


so that $-\boldsymbol{v} q \boldsymbol{v}$ leaves the scalar part 1 of $q$ invariant, and converts the vector part “i $+\mathbf{j}-\mathbf{k}$ ” of $q$ into "i-2j”. From equation (9), the dual-quaternions corresponding to unit pure semi-quaternions "i $+\mathbf{j}-\mathbf{k}$ ", "i $-2 \mathbf{j} "$ can be given, respectively, by $\boldsymbol{Q}=\boldsymbol{i}+\boldsymbol{j} \varepsilon-\boldsymbol{k} \varepsilon$ and $\boldsymbol{Q}^{\prime}=\boldsymbol{i}-2 \boldsymbol{j} \varepsilon$. By the Study map, the lines corresponding to unit pure dual-quaternions $\boldsymbol{Q}, \boldsymbol{Q}^{\prime}$ intersects the plane $x=1$ of $\mathbb{R}^{3}$ at the points, respectively, $Q=(1,1,1), Q^{\prime}=(1,0,-2)$. It is easily checked that the point $Q^{\prime}=(1,0,-2) \in \mathbb{R}^{3}$ is a reflection of the point $Q=(1,1,1) \in \mathbb{R}^{3}$ through the point $Q=(1,1 / 2,-1 / 2) \in \mathbb{R}^{3}$. The same result can be given straightforward by using the item 1 in Proposition 7.

\section{References}

[1] Ata E. and Yayli Y., Dual Unitary Matrices and Unit Dual Quaternions, Diff. Geom. Dyn. Syst. 10 (2008) 1-12.

[2] Bekar M. and Yayli Y., Involutions of Complexified Quaternions and Split Quaternions, Adv. Appl. Clifford Algebras 23 (2013) 283-299.

[3] Bekar M. and Yayli Y., Dual-Quaternion Involutions and Anti-Involutions, Adv. Appl. Clifford Algebras 23 (2013) 577-592.

[4] Bekar M. and Yayli Y., Involutions in Dual Split-Quaternions, Adv. Appl. Clifford Algebras 23 (2013), doi: 10.1007/s00006-015-0624-z.

[5] Ell T. and Sangwine S., Quaternion Involutions and Anti-Involutions, Comput. Math. Appl. 53 (2007) 137-143.

[6] Feiten W., Lang M. and Hirche S., Rigid Motion Estimation using Mixtures of Projected Gaussians, In: 16th International Conference on Information Fusion-(FUSION 2013).

[7] Hahn A., Clifford Algebras, and Arithmetic Witt Groups, Springer, New York 1994.

[8] Hamilton W., On a New Species of Imaginary Quantities Connected with the Theory of Quaternions, Proc. Roy. Irish Acad. 2 (1844) 424-434.

[9] Jafari M. and Yayli Y., Hamilton Opeators and Generalized Quaternions, In: 8th Congress of Geometry (2010).

[10] Kuipers J., Quaternions and Rotation Sequences, Princeton University Press, Princeton 1999, See also: Kuipers J., Quaternions and Rotation Sequences, Geom. Integrability \& Quantization 1 (2000) 127-143.

[11] Rosenfeld B., Geometry of Lie Groups, Kluwer, Dordrecht 1997.

[12] Ward J., Quaternions and Cayley Algebras and Applications, Kluwer, Dordrecht 1996. 
Received 22 February 2016

\author{
Murat Bekar \\ Department of Mathematics and Computer Sciences \\ University of Necmettin Erbakan \\ Konya 42090 \\ TURKEY \\ E-mail address: mbekar@konya.edu.tr \\ Yusuf Yayli \\ Department of Mathematics \\ University of Ankara \\ Ankara 06100 \\ TURKEY \\ E-mail address: yayliescience.ankara.edu.tr
}

